\title{
Macedonian Medical Master of Science Theses in Public Health (MScPH) Defended in 2012
}

\author{
Macedonian Journal of Medical Sciences* \\ Institute of Immunobiology and Human Genetics, Faculty of Medicine, Ss. Cyril and Methodius University of Skopje, Skopje, \\ Republic of Macedonia
}

\begin{abstract}
Citation: Macedonian Journal of Medical Sciences. Macedonian Medical Master of Science Theses in Public Health (MScPH)

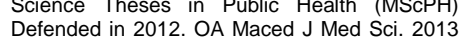
Defended in 2012. OA Maced J Med Sci. 2013 Dec
http://dx.doi.org/10.3889/oamjms.2013.028 Key words: Master of Science in Public Health (MScPH); Public Health; Medical research; Republic of Macedonia.

"Correspondence: Macedonian Journal of Medical Sciences. Institute of Immunobiolog and Human Genetics, Faculty of Medicine, Ss. Cyril and Methodius University of Skopje, Republic of Macedonia. 50 Divizija No 16, PO Box 60, 1109 Skopje, Republic of Macedonia. Telephone: +38923110556 . Telefax: +389 3110558. EMail:mjms@ukim.edu.mk

Received: 11-Nov-2013; Revised: 20-Nov2013; Accepted: 02-Dec-2013; Online first: 12-Dec-2013

Copyright: () 2013 Macedonian Journal of Medical Sciences. This is an open-access article distributed under the terms of the Creative Commons Attribution License, which permits unrestricted use, distribution, and reproduction in any medium, provided the original author and source are credited.

Competing Interests: The authors have declared that no competing interests exist.
\end{abstract}

\begin{abstract}
We present English abstracts of MSc theses in Public Health (MScPH) defended in 2012 at the Faculty of Medicine, Ss. Cyril and Methodius University of Skopje, Republic of Macedonia. English summaries are published as they are translated by authors and included in the final version of defended MScPH. Macedonian Medical Master of Science theses are deposited in the Central Medical Library and National and University Library "St. Kliment Ohridski" in Skopje.
\end{abstract}

At the Faculty of Medicine in Skopje 17 MSc theses in Public Health there were defended in 2012 five of them are not deposited in the Central Medical Library. All deposited MScPH have abstracts in Macedonian and English language, with key words, mentors and co mentors.

Editorial Board does not take any responsibility either for the content, nor the quality of the abstracts. Primary responsibility for the quality of the MSc theses belongs to the mentors, to the institutions they are representing, and to the Vice-Dean of science.

Defended MScPH can be cited as they are published in this and in the previous reports.
Zorica Vrchkovska. Influence of style living on the population health in urban and rural parts of the municipality Bitola [MScPH thesis]. Skopje, Republic of Macedonia: Centre for Public Health, Faculty of Medicine, Ss Cyril and Methodius University of Skopje; 2013. OA Maced J Med Sci. 2013 Dec 15; 1(1):148-159.

Not available in the Central Medical Library.

Mentor: Prof. Dr. Elena Gjoshevska.

Defended: 11.01.2012.
Valentina Andonova. International Guide for Health. Laboratory capacities for detection of chemical risks in public health [MScPH thesis]. Skopje, Republic of Macedonia: Centre for Public Health, Faculty of Medicine, Ss Cyril and Methodius University of Skopje; 2013. OA Maced J Med Sci. 2013 Dec 15; 1(1):148-159.

Public health with its wide range of organized activities has international obligations, such as procedures in case of emergency public - health circumstances, concerning not only the country but having an international significance, as well. The 
application of the health measures from the International Health Regulations (IHR) include readiness in case of emergency, taking measures in order to prevent, protect, and control, as well as securing response in case of international spread of disease in a way that is in agreement with public health risk. It is also ready to react globally to any natural catastrophe, accidental or purposeful release of chemical hazards, etc.

The main purpose of this Master's thesis is to determine the readiness of the laboratory institutions in the Republic of Macedonia to evaluate the chemical risks of public-health character which are subject of interest of IHR. To obtain all these, it was necessary to examine the strategic readiness of the laboratory institutions in managing chemical risks of public-health character through examining their competency as far as the staff is concerned, premises, equipment and methods used to determine chemical risk, as well as application of quality standards such as ISO 17025, ISO 15189 and Good laboratory practice covered by IHR.

Ten laboratories were included in the research, and the data obtained was due to the information collected from the 87 interviewed employees in these laboratories. They all were with higher academic education. The first survey questionnaire was made by $\mathrm{WHO}$ and it concerns strategic readiness of the country's Chemical safety capacity, whereas the second survey questionnaire was based on legal documents i.e. the Regulations for the principles of Good laboratory practice. The research results showed that the existing chemical and bio-chemical laboratories in the Republic of Macedonia, which are possible participants in the system of detection and management of the chemical risks of public health character, do not fully meet the quality and Good laboratory practice standards.

The general conclusion from this research is that the laboratory institutions in the Republic of Macedonia are not ready for the evaluation of the chemical risks of public-health character which are subject of interest of IHR. They are also not ready to meet IHR requirements concerning the management of the chemical risk of public-health character.

Key words: IHR; chemical hazard; quality standards; Good laboratory practice; ISO 17025; ISO 15189.

Mentor: Prof. Dr. Marija Krstevska.

Comentor: Prof. Dr. Dragan Gjorgjev.

Defended: 26.3.2012.

Mirjana Dimovska. Level of environmental health awareness among children and youth and their impact on the policy in this area [MScPH thesis]. Skopje, Republic of Macedonia: Centre for Public Health, Faculty of Medicine, Ss Cyril and
Methodius University of Skopje; 2013. OA Maced J Med Sci. 2013 Dec 15; 1(1):148-159.

Introduction: Several studies show that the risks from the environment are responsible for $25-35 \%$ of the global burden of disease. The population of children and youth in the Republic according to the State Statistical Office is $31.2 \%$ of the total population in RM. In 2004 WHO developed CEHAPE (Children's Environment and Health Action Plan for Europe) in order to reduce the burden of diseases ill children attributable to environmental factors. The views of young people and their inclusion should be a fundamental part of the development plans of each country.

Main objective: The main objective of this paper is to identify the needs, priorities, level of awareness, attitudes and beliefs of children and young people in terms of health and environment, to develop targeted campaigns to communicate and promote Nation al Environmental Health Action Plan (NEHAP) and CEHAPE.

Material and methods: Survey represents Crosssectional study. For the purposes of the survey two survey questionnaires were used, adapted, revised and translated from English into Macedonian and Albanian. The first questionnaire "Healthy environments, healthy child. Creating a better future, and a second questionnaire "Environmental Review" were utilized. The field activities were conducted in terms to provide information about the activities of all involved and interested stakeholders. The survey was done among 1359 respondents aged 12 to 14 years in primary school and 15-18 years, high school students, from the 6 statistic regions in the RM. The questionnaire for students was disseminated in selected primary and secondary schools, and accomplished during the class hour. The second questionnaire was filled out by selected school teams (managers, teachers and support staff). Statistical analysis of data performed with SPSS 17.0 and Statistica 8.0. For testing the statistical significance of differences in the distribution of responses is cross tabulation and Chi-square test. Applied statistical significance of $\mathrm{p}<0.05$ (results are considered "statistically significant"). Results with a value of p>0.05 are considered as "the absence of statistical significance."

Results: $53.64 \%$ of the respondents were female while $46.36 \%$ are male, aged 12 to 18 years. The majority were Macedonians $64.02 \%$, followed by $29.21 \%$ Albanians, $3.31 \%$ were Turks, $1.25 \%$ Serbs and others (Roma, Vlachs, Bosniaks and Torbesh) comprised $2.21 \%$. Most respondents classified their health as either very good or quite good. There is a significant difference in terms of barriers to a healthy lifestyle among the respondents from urban and rural areas as well as primary and secondary schools. Most respondents were aware of the environmental impact on their health. Television and the Internet are the 
main sources of information on the environmental impact on their health. The main priorities that significantly affect their health respondents considered the immediate environment, then drinking water quality and safety and other topics. Most respondents were able to identify certain conditions in the environment as a problem, but not to suggest ideas for improvement. Respondents from the urban area, high school aged and males are less motivated and interested to be included in further activities. The results of the second survey showed that current state in elementary and secondary schools partly correspond to the actual needs for environmental protection.

Recommendations: Creating a better and healthier environment requires a strong commitment and involvement of all actors in society responsible for ensuring good health and healthy environment: Government and NCOs, local government and community in its entirety. Active involvement of children and young people is the fundament in children's environmental and health policy making process.

Key words: children and youth; priorities; environment and health; school.

Mentor: Prof. Dr. Dragan Gjorgjev.

Defended: 27.03.2012.

Aleksandar Jovanovski.The knowledge, positions and actions of the medical students on the factors of risk for HIV/AIDS and estimation of their stigma to HIV positive patients [MScPH thesis]. Skopje, Republic of Macedonia: Centre for Public Health, Faculty of Medicine, Ss Cyril and Methodius University of Skopje; 2013. OA Maced J Med Sci. 2013 Dec 15; 1(1):148-159.

Introduction. One of the bigger concerns of the public health in the 21st century is the pandemic of HIV/ AIDS, due to complexity of the issue, global spreading, different ways of transmission, of which the most common are injecting drugs (intravenously) and sexual transmission, followed by huge and impoverishing medical as well as other direct and indirect expenses and suffering from other concomitant diseases - comorbidity, such as tuberculosis, respiratory infections and malignant diseases. AIDS patients need care that might be very expensive. It is considered that 3.5 million patients will receive antiretroviral therapy (ART) in the countries with low and middle income by the end of year 2011, through various programs for treatment co-financed by the Global Fund. It is estimated that during the period of 2011 - 2020, the required funds in these countries for the aforementioned programs will amount to 14.2 billion dollars; however, this investment will contribute to save even 18.5 million life years.
The main aim of this study was to define the knowledge, attitudes and practices (KAP) of the students of medicine toward the risk factors for transmission of HIV infection and evaluation of the stigmatization to HIV positive patients, as well as to give recommendations for their changes and diminishing the stigmatization to HIV positive patients. General and specific objectives were related to assessing knowledge, attitudes and practices of students of medicine to the risk factors for transmission of HIV infection and stigmatization of students of medicine to HIV positive patients who belong to marginalized subgroups according to demographic characteristics (sex, age and place of residence), socio-economic characteristics and year of study. Five hypotheses were set for realization of the defined goals.

Materials and methods. This is a prospective crosssectional study analyzing the knowledge, attitudes and practices of students of medicine towards risk factors for HIV transmission and evaluating the degree of stigmatization to HIV positive patients, by the students of medicine at the Faculty of Medicine, Ss Cyril and Methodius University, Skopje, where the study was conducted in the period of May-June 2011. The investigation is a part of a multi - center study conducted in 5 countries - Mongolia, Georgia, Ukraine, USA and Macedonia. A sample of 330 students of medicine, equally distributed in all years of studies took part. A unified and specially designed questionnaire as an instrument of the investigation was applied. Statistical analysis was done using the SPSS 16.0. program and standard statistical methods. All statistical tests have been processed by use of required statistical significance which was defined as a $P$ value $<0.05$.

Results and discussion. Of the total number of examined students of medicine (330), 207 (62.73\%) were females and 123 (37.27\%) males; the youngest participant was 18 years of age and the oldest 26 years of age. As expected, $91.8 \%$ of students are not married, and $67 \%$ do not live with husband/wife or permanent sexual partner. $47.3 \%$ of students came from another town, $11.5 \%$ of students came from village, $4(1.2 \%)$ students came from other countries, the remaining percent was from Skopje, the largest percent of the participants (35.5\%) live with their parents. Almost half of the participants $(45.8 \%)$ have relatively good socio-economic status. As for their future specialization, $44.5 \%$ students will choose surgery, $21.8 \%$ internal medicine, only $8.8 \%$ will choose preventive medicine, and $24.9 \%$ will choose other medical branches. The results of the study have completely confirmed the set working hypothesis. Differences in knowledge, attitudes, and practices of students of medicine for HIV infection, depending on sex, age and place of residence, exist. Stigmatization to HIV positive patients is present in students in any year of studies, with higher degree of stigmatization in students attending clinical years, compared to 
students attending preclinical years of studies. Place of residence and community in which students of medicine live have influence on their stigmatization. The students are more concerned about that they may be infected by HIV/AIDS if they take care of a HIV positive patient than about their personal risk behaviour. The results obtained were analyzed and compared with results from studies performed worldwide. A number of studies presented similar results as ours concerning knowledge, attitudes and practices of students of medicine to the risk factors for transmission of HIV infection and stigma towards HIV positive patients and members of marginalized subgroups.

Conclusion. Differences in knowledge, attitudes, and practices of students of medicine regarding the HIV infection, including the present stigmatization toward HIV positive patients and HIV negative members of marginalized sub-groups, imply the need of appropriate medical education of students of medicine throughout the study process, as well as the modifications in curriculum of the Faculty of Medicine in Skopje. The students of medicine are future doctors of medicine, and therefore, stigmatization must not exist.

Key words: knowledge; attitudes; practices; stigmatization; HIVIAIDS; students of medicine.

Mentor: Prof. Dr. Fimka Tozija.

Defended: 28.03.2012.

Iskra Garazova. Family function in the alcohol addictions as a public health problem [MScPH thesis]. Skopje, Republic of Macedonia: Centre for Public Health, Faculty of Medicine, Ss Cyril and Methodius University of Skopje; 2013. OA Maced J Med Sci. 2013 Dec 15; 1(1):148-159.

Not available in the Central Medical library.

Mentor: Prof. Dr. Rozalinda Isjanovska.

Defended: 29.03.2012.

Ana Karapejovska. Estimation of the grade of preparedness for accreditation of the Border Inspection Department for the ISO/IEC 17020, of the Agency for Food Safety, Veterinary and Phytosanitary Policy from the public health aspect [MScPH thesis]. Skopje, Republic of Macedonia: Centre for Public Health, Faculty of Medicine, Ss Cyril and Methodius University of Skopje; 2013. OA Maced J Med Sci. 2013 Dec 15; 1(1):148-159.

Introduction: One of the requirements, namely midterm priorities according to Chapter 3.12 of the National Programme for Adoption of EU Acquis - Food safety, Veterinary and Phytosanitary policy - which Food and Veterinary Agency must fulfil, is accredited inspection body in accordance with ISO/IEC 17020 -
General criteria for the operation of various types of bodies performing inspection.

Main objective: Assessment of the preparedness for accreditation of Food and Veterinary Agency, precisely Border Inspection Department for ISO/IEC 17020 standard.

Material and methods: A cross-sectional was conducted. For the purposes of the study, control list was prepared for the analysis of the current, as well as the status after the study was finished, of the Border Inspection Department regarding preparedness for fulfilment of the requirements according to ISO/IEC 17020 standard.

Results: 1. Administrative requirements are completely fulfilled, but regarding human resources, equipment and spatial-infrastructure capacities, requirements are partially met. 2 . The required procedures, identified during the study, as well as Policy and Quality Manual are submitted for admission. 3. Official veterinarians and state food inspectors on border posts are not trained for ISO/IEC 17020 standard.

Recommendations: 1 . The number of the staff performing official controls on border inspection posts should be increased. 2. New equipment should be supplied and the equipment should be calibrated. 3 . Strengthening of the spatial infrastructure capacities. 4. Training of officials in Border Inspection Department should be conducted regarding ISO/IEC 17020 requirements.

Key words: Food and Veterinary Agency; Border Inspection Department; ISO/IEC 17020, accreditation.

Mentor: Prof. Dr. Mihail Kochubovski.

Defended: 06.04.2012.

Zharko Stojmanovski. Public health aspects of brucellosis in the Republic of Macedonia [MScPH thesis]. Skopje, Republic of Macedonia: Centre for Public Health, Faculty of Medicine, Ss Cyril and Methodius University of Skopje; 2013. OA Maced J Med Sci. 2013 Dec 15; 1(1):148-159.

Introduction: Brucellosis in Republic of Macedonia is one of the main zoonosis among population and serious health problem. In 2008 in Republic of Macedonia the strategy of inhibition and eradication of brucellosis among sheep's and goats is changed and for the first time sheep and goats vaccination against brucellosis is introduced and implemented.

Main goals: Incidence rate to be determined, prevalence rate and development tendency of infected by brucellosis in $R$. Macedonia in period from 01.01.1999 to 31.12.2009, registration and analyses of the epidemiological characteristic's of the infected from brucellosis according to gender, age, territorial distribution among regions, the estimation of the rate of incidence of brucellosis among sheep and goats 
before and after vaccination during the probing period, a correlation between the number of infected sheep and goats which are registered in the authorities of the public administration and public health institutions in terms of brucellosis in R. Macedonia and adequate suggestions and activities for improving the control and inhibition of brucellosis in R. Macedonia have to be specified.

Materials and methods: A descriptive-analytical survey was conveyed where all information about distribution and development tendency of brucellosis among population, sheep and goats are shown, defined and given. The information about infected people from brucellosis were found by registration of several cases as well as monthly and annual reports for infectious diseases from the Institute tor Public Health of R. Macedonia. Information for infected sheep and goats are taken from the Food and Veterinary Agency. Information for existing programs and preventing activities for protection from brucellosis infection were found by conducting surveys, questionnaires.

Results: According to the survey the highest number of incidence of human brucellosis in R. Macedonia is found in year 2008, the number is 23.94/100,000 people, and the lowest number of infected people was in year 2009, about $13.99 / 100,000$ people. The growing in R. Macedonia is falling down. Males (69\%) are dominant tendency of human brucellosis thought years and they are statistically more dominant than the females (31\%). Brucellosis is notably higher among the group of people at the age of 60 and more, than it is $17.85 \%$ from the whole number of infected people, and at the age of $0-9$ years, the number of infected people is $6.86 \%$ and also the presence of the infection, illness. The highest rate of incidence of brucellosis among sheep and goats is registered in 2007 and the number is 2010/100,000 sheep and goats, and the lowest rate is in 2000 , and the number is $161 / 100,000$ sheep and goats. Periodical prevalence of brucellosis among sheep and goats before introduction of vaccination is 6580/100,000 sheep and goats, and after the vaccination $3125 / 100,000$ sheep and goats $(p<0.05)$. There is light negative correlation between the number of infected people from brucellosis and the number of infected sheep and goats $(6=-0.35)$.

Conclusion: Falling of the rate of incidence and prevalence of human and animal brucellosis or its eventual eradication a i-disciplinary approach is inevitable and multi sector collaboration between all relevant institutions in the fields of public health service and veterinary health service at all levels and rates. The most effective method for prevention of human brucellosis is eradication of animal brucellosis. The success of control programs will be primary estimated with lowering of human cases of brucellosis.
Key words: Human brucellosis; sheep and goats' brucellosis; brucellosis vaccination; multisector collaboration.

Mentor: High sci. coll. Dr. Milka Zdravkovska.

Defended: 12.04.2012.

Violeta Jakimovska. The level of ocular pressure as a factor of risk for the glaucoma disease through public-health action for early detection of the disease [MScPH thesis]. Skopje, Republic of Macedonia: Centre for Public Health, Faculty of Medicine, Ss Cyril and Methodius University of Skopje; 2013. OA Maced J Med Sci. 2013 Dec 15; 1(1):148-159.

Introduction: The World Health Organisation estimates that there are 45 million blind people and 180 million people with impaired sight, most of which are living in Third World countries, where the blindness rate reaches up to $1-2 \%$ of the total population. Because glaucoma takes second place on the list of ophthalmological diseases with the highest morbidity, it has great significance in the world of medicine as well is finding an efficient way to cure this disease, thus decreasing the percentage of blindness that may be caused by glaucoma. Early discovery of the illness is crucial for prompt treatment, improving the patients' lives and stopping blindness, which is the last stage of the illness. The Association of Ophthalmologists of Macedonia took part in celebrating the 2011 World Week of Fighting against Glaucoma with a mass event which included measuring eye pressure - the number one cause of contracting glaucoma - in 7 cities in the Republic of Macedonia.

Main Goal: Screening of population with elevated eye pressure and other risk-inducing elements connected to their life style, which are responsible for the emergence of glaucoma, its early discovery and the prevention of other glaucomatous impairments upon the eye nerve.

Materials and Methods: This study is designed as retrospectively transversal, where the processed data has been gathered by interviewing volunteers in the event, led by the motto "Do not lose sight of your family", conducted in the month of March 2011 in the cities of Skopje, Bitola, Ohrid, Shtip, Gostivar, Tetovo and Kumanovo. The study analyses data gathered from 1306 volunteers as well as recorded eye pressure after each interview. The volunteers were then divided into three groups.

1. The first group consists of 159 volunteers who had already been diagnosed with glaucoma and who were advised to regularly take their medication and regularly visit their ophthalmologist.

2. The second group consists of III volunteers who were diagnosed with elevated eye pressure and who 
were advised to regularly visit their ophthalmologist for further examinations.

3. The third group consists of 1036 volunteers who had never had glaucoma, nor were they diagnosed with elevated eye pressure during the event.

Stat Soft Statistica 10 was used for processing all of the data. The statistical analysis was done by using the standard statistical methods for levels of significance of $\mathrm{p}<0.05$ and high that a history with glaucoma within a family is a factor which increases the risk of elevated eye pressure by 3.5 , myopia by 1.5 and high levels of sugar in the blood doubles the risk of having high eye pressure. Lifestyle proved to play a big role in differentiating subjects with high blood pressure since most of them smoke, consume alcohol and feed irregularly. Living in the city is a statistically significant risk factor which increases the risk of elevated eye pressure by 1.5 times, as well as ages above 60 which increase the risk of elevated eye pressure by the same amount. In terms of being informed, the subjects who had already been diagnosed with glaucoma are far more informed than others. The majority of volunteer subjects consist mainly of men above the age of 60 . Regarding their employment status, most of the subjects are retired, while there is a small number of employed and unemployed as well. It has been confirmed that everyday intake of other medicine does not influence registering high blood pressure and that there is no difference between subjects who take medicine and those who do not.

Conclusion: The public health event, meant for discovering elevated eye pressure within the volunteer subjects, has been realized. Other risk factors which indirectly influence the elevation of eye pressure and glaucoma, which are also connected to the subject's life style such as food, alcohol and smoking, have also been confirmed. The subjects who had already been diagnosed with glaucoma have changed their life style, thus some of them have stopped smoking, and started feeding regularly, unlike the second and third group subjects, who had not been diagnosed with the illness. Finally, risk factors such as one's family glaucoma history, myopia, diabetes and elevated blood pressure as well as smoking, consuming alcohol and irregular food intake have proved plausible in their influence, where one's location of residence and other medicine intake have not.

Key words: glaucoma; blindness; risk factors; elevated eye pressure; screening; public health event; prevention.

Mentor: Prof. Dr. Rosalinda Isjanoska.

Defended: 14.06.2012.

Katerina Venovska. Evaluation of the works of the medical doctors in primary health protection which started with additional education in the family medicine in the Educative Center in Skopje with the accent on the control of patient with artery hypertension [MScPH thesis]. Skopje, Republic of Macedonia: Centre for Public Health, Faculty of Medicine, Ss Cyril and Methodius University of Skopje; 2013. OA Maced J Med Sci. 2013 Dec 15; 1(1):148-159.

Introduction: All Chosen Doctors (doctors) in the Primary Health Care (PHC) in the Republic of Macedonia (RM), every year sign Contracts with the Health Insurance Fund of Macedonia which pays them a capitation fee based on the number of registered patients and a point multiplier for the different age groups of patients. After the Ministry of Health (MOH) developed the long term Health Strategy 2020 (HS 2020) adopted by the Government of RM in 2006, amended in 2007 and the Family Medicine implementation Strategy (FMIS), the Family Medicine Specialization was initiated in October 2009 and the reeducation for the doctors working in General Medicine practices (General Practitioners/ GPs) in PHC started in February 2011.

The Main Goal of this study is evaluation of the work of GPs in the general medicine practices that have first started with the FM reeducation activities, their knowledge and skills as implemented in practice and documentation of the advice and recommendations given to the patients in practice in the patient's health records. The Specific Objectives are: a) evaluation of the management of patients according to the purpose of the visit, the investigations carried out, referrals to specialists and the most common diagnosis, b) evaluation of the management of patients with hypertension according to the patients' history records, the clinical examination results, the documented advice to the patients as recorded in their health cards and the most commonly prescribed medicines to patients with hypertension; and c) prepare a profile of most motivated doctors that first started with the FM reeducation activities and describe their practices.

Material and Methods: The study was carried out in the period from April until June 2011 cove ring 30 GPs from general medicine practices in 5 health regions in RM (Skopje, Veles, Kumanovo, Tetovo and Gostivar) that were undergoing FM reeducation activities provided data and completed specially designed questionnaires for the management of 10 patients (interviewees) aged between 25 and 65 years, out of which at least 8 were already diagnosed with arterial hypertension hence the study covered 300 cases in total.

The Results are presented in accordance to the location of the practices (urban and rural areas) and the length of the work experience of chosen doctors in PHC: Group 1 (less then 7 years); Group 2 (7-14 years); and Group 3 (over 14 years). The Study responded to the defined aims of the research, however, except the general hypotheses, only some 
of the specific hypotheses were confirmed. With regard to the General Hypotheses from the analyzed elements of the hygienic-dietetic regime of patients with hypertension, all three groups of doctors in average to half of the patients gave advice and registered in their health re cord other dietetic measures and decreasing of body weight, whereas the difference between the three groups of doctors for the second parameter is significant (Chi-square $=15,85$ $d f=4 \quad p=0,0032)$. Among a sign ificantly lower percentage of patients in urban and rural areas the advice to cease smoking (34\% vs $11,8 \%$ - Chisquare $=19,65 \mathrm{df}=2 \mathrm{p}=0,000054)$ and to decrease alcohol intake $(11,2 \%$ vs $9,7 \%$ - Ch i-square $=6,52$ $d f=2 \quad p=0,038)$ are registered, however, more frequently among doctors in the urban areas. Among the patients with hypertension the doctors in rural practices were measuring and registering the pulse data significantly more frequently (Chi -square $=14,76$ $\mathrm{df}=1 \mathrm{p}=0,00012$ ) and more then half of the doctors were controlling and registering the blood pressure data during each visit to their practice. The doctors were maintaining a registry in average for less then half of their patients with hypertension, whereas the differences among the chosen doctors in urban and rural practices $(24,9 \%$ vs $69,7 \%)$ are highly significant (Chi-square $=55,68 \mathrm{df}=1 \mathrm{p}<0,001$ ). The doctors with over 14 years working experience were referring less to specialists $(47,5 \%$ patients were not referred), compared with the doctors with less then 14 years working experience (didn't refer $45.7 \%$ patients), but the difference is not statistically significant $(p=0,79)$. The interventions like ears washing, foreign objects removal, inhalations and others, were not performed among anyone of the patients in rural practices, and only a small number of interventions were performed among eight patients in total in urban practices, which presents a significant difference. With regard to the degree of Minimal Diagnostic Program (MOP) implementation and data registration among patients with hypertension in accordance to the length of the work experience in $\mathrm{PHC}$, show a significant distinction among all three groups of doctors (Chi-square $=11,68$ $d f=4 p=0,02)$. The highest percentage of patients of the doctors of Group 1 (64\%), have completed the full diagnostic program that was registered in the patients' medical documentation. There is an insignificant higher registering of data for the lipid status among doctors of Group $2+$ Group 3, for the smoking status among doctors of Group I, and for the pulse measurement among doctors of Group 3. With regard to the blood pressure measurement data, the difference in patient distribution is significant among doctors of Group 1 and of Group 3 (Chi-square=1I,99 $d f=3 p=0,007)$. The tested differences in the frequency of the blood pressure measurements show a statistically significant distinction among all three groups of doctors with diverse length of working experience in the PHC (Chi-square=26,98 $\mathrm{df}=6$ $p=0,00071)$. The highest percentage of patients among all three groups of doctors were getting one medicine only (59\% vs $57 \%$ vs $45 \%$ ), a considerably lower number of patients were getting fixed combinations of medicines (op, $16 \%$ vs $22 \%$ vs $23 \%$ ) most frequently being prescribed by the doctors of Group 3, and the rest were getting free combinations of medicines for the regulation of high blood pressure ( $26 \%$ vs $20 \%$ vs $32 \%$ ). The difference is not statistically significant (Chi-square $=1,52 \mathrm{df}=\mathrm{l} p=0,22)$.

Conclusions: The Chosen Doctors should: follow up with their patients in a more systematic way, to improve the maintenance of registry for patients with hypertension, to provide advice for the ceasing of smoking and practicing of various dietetic measures, more frequently, and to increase the frequency of interventions performed in PHC practices, especially in rural areas. The Study results will serve as a platform for comparison with the results of some other studies in this field in future and for the evaluation of the implementation success of the FM concept in the $\mathrm{RM}$ and could be also used as a guidance by the FMEC, to strengthen educational activities within the frames of the reeducation programme for doctors, especially in the context for improving of the management of patients with arterial hypertension and registering of their work.

Key words: primary health care; family medicine; reeducation; Chosen Doctors; evaluation; patients; hypertension; management.

Mentor: Prof. Dr. Doncho Donev.

Defended: 18.06.2012.

Gjorgji Pecirov. Colorectal carcinoma in the Republic of Macedonia - estimation of the risk for the colorectal carcinoma in the Strumica regison [MScPH thesis]. Skopje, Republic of Macedonia: Centre for Public Health, Faculty of Medicine, Ss Cyril and Methodius University of Skopje; 2013. OA Maced J Med Sci. 2013 Dec 15; 1(1):148-159.

Not available in the Central Medical Library.

Mentor: Prof. Dr. Doncho Donev.

Defended: 28.06.2012.

Mahir Ziberi. Estimation of dietetic income and attitudes for nourishing in the rural fortifications along the Vardar River [MScPH thesis]. Skopje, Republic of Macedonia: Centre for Public Health, Faculty of Medicine, Ss Cyril and Methodius University of Skopje; 2013. OA Maced J Med Sci. 2013 Dec 15; 1(1):148-159.

Chemical and physical hazards emitted from any source can lead to contamination of all media in the environment, but usually ends their deposition in surface water and land. By here it can be transferred to groundwater and than taken from plants and animals and thus enter the food chain where it 
occurred and can be accumulated. River Vardar is the recipient of various types mostly untreated wastewater and thus represents a potential risk for pollution of groundwater, drinking water, flora, fauna and human at the top of the food chain. As contaminants occurring: residues or heavy metals, pesticide residues, mycotoxins, radionucleotides, antibiotics, POPsi and other organic substances. Their presence in food can cause the effects of acute or chronic food poisoning. Several factors determine the likelihood and severity of health effects, including the number and characteristics of contaminated agents, the nature of the food habits or consumption. The main objective or this master thesis is to determine the intake of food and dietary habits of the rural population of different ethnicities in the context of possible contamination from River Vardar in order proposing a public health measures and activities based on the obtained results. Population group involved in the research are residents from Zelenikovo and Zelino. All respondents living In villages near the river Vardar, and who use it to irrigate their fields which produce food for home use. Examined groups are distinguished by one characteristic ethnicity. There is a difference in nutritional intake of the studied population groups or different ethnicities but it is not statistically significant. 24-hour dietary analysis confirmed that average daily intake among respondents in Zelino is $1735 \mathrm{kcal}$, most of which represents the group or cereals and their products $(890 \mathrm{kcal}$ or $51 \%)$ and average daily intake among respondents from Zelenikovo is 1853 $\mathrm{kcal}$, most accounts or the group of cereals and their products $(844 \mathrm{kcal}$ or $45 \%)$. There is no statistical significant difference in intake or contaminants in food in terms of consumed products that are near the river Vardar as a potential contaminant flow after the towns of Tetovo and Skopje. The statistical analyses of obtained data or the health risk were founded normal range of daily intake for lead and cadmium among both men and women in Zelino and Zelenikovo. The estimated daily Intake of $\mathrm{Pb}$ and $\mathrm{Cd}$ in this study are in the range of those values recommended by $\mathrm{FAO} /$ $\mathrm{WHO}$, but in case of increasing the intake or intake of recommended daily intake or fruits and vegetables (> $600 \mathrm{~g}$ ) very likely existing content or residues of cadmium and lead in home produced vegetables and fruits will pose a health hazard to consumers in both regions. The obtained results will assist in developing public health recommendations or guidelines for diet for specific population groups in the planning or nutrition in rural areas.

Key words: dietary assessment; chemical contamination; Vardar River; rural population, Zelino, Zelenikovo; risk; public health recommendations.

Mentor: Sci. coll. Dr. Vladimir Kendrovski.

Defended: 10.07.2012.

Rahela Stefanovska. Realization of the rights and the health of the patients from the quality of the services in the rpivate health institutions in Skopje [MScPH thesis]. Skopje, Republic of Macedonia: Centre for Public Health, Faculty of Medicine, Ss Cyril and Methodius University of Skopje; 2013. OA Maced J Med Sci. 2013 Dec 15; 1(1):148-159.

Not available in the Central Medical Library.

Mentor: Prof. Dr. Mome Spasovski.

Defended: 15.11.2012.

Ejub Gjulsha. Public health aspects of some comoribities in the patients on hemodialysis in the Department of Nephrology, Struga [MScPH thesis]. Skopje, Republic of Macedonia: Centre for Public Health, Faculty of Medicine, Ss Cyril and Methodius University of Skopje; 2013. OA Maced J Med Sci. 2013 Dec 15; 1(1):148-159.

Not available in the Central Medical Library.

Mentor: Sci. coll. Dr. Mila Zdravkovska.

Defended: 28.11.2012.

Ljubica Todorova. Evaluation of the effects of introducing the model of payment - Diagnostic similar groups in the General Hospital Kochani [MScPH thesis]. Skopje, Republic of Macedonia: Centre for Public Health, Faculty of Medicine, Ss Cyril and Methodius University of Skopje; 2013. OA Maced J Med Sci. 2013 Dec 15; 1(1):148-159.

Introduction: In 2009 in the Republic of Macedonia there has been an implementation of a new method of payment in the hospitals, which includes application or financing based on mixed cases, where the outcome results of the hospital are measured as Diagnostic Related Groups (DRG). This has transformed the current payment system in the hospitals based on historic financial distribution into a payment system based on the outcome result measured with DRG.

Main objective of the research is evaluation of the effects of the intervention implementation or the DRG model of payment in the Public Health Institution General Hospital Kochani, with analysis of the clinical performances of the hosp ital before and after the implementation of DRG. For the realization or the general and specific objectives set and tested five hypotheses research.

Materials and methods: The study was designed as a retrospective cross sectional study (cross-sectional). It was applied a public health approach to evaluation of the effects of the intervention - introducing a new method of payment per DRG for hospital treated people in 2009. The study analyzed data on patients in the General Hospital Kochani in the period 20072008 , before the introduction or the DRG and in the period 2009 - 2010 after the introduction of DRG. Data on method of payment according to the services 
performed arc taken from local databases of health statistics for 2007 - 2008, and payment under the DRG data are used by the central database of the DRG grouper fund 2009 - 2010 Kochani hospital. The compared aspects are: number of hospital inpatients; average duration of hospitalization (length of hospital stay); number of readmitted patients for reoperation for the same diagnosis within one month after discharge; number of readmitted patients for the same diagnosis within three months after discharge; number or referrals to other hospitals; number or intra-hospital infections; hospital costs.

Results: The number of the hospitalized patients has decreased with the implementation of the DRG model of payment. Index of 93.1 for Surgical Ward was 91.8 for the Internal Medicine Ward, 84 for the Children's Ward and 80.6 for the Neuropsychiatric Ward. Also reduces the number of hospital days after the introduction of the DRG with the following indexes: 90 for the Internal Medicine Ward, 88.4 for the Gynecology and Obstetric Ward, 83.4 for the Children's Ward, 74.8 for the Surgical Ward and 74.6 for the Neuropsychiatric Ward. The number of references to the tertiary level is reduced by the introduction of the DRG, with an index of 87.3 Surgical Ward, 53.2 Gynecology and Obstetric Ward. Length or hospital stay mostly reduces Surgical Ward, Neuropsychiatric Ward and Children's Ward.

Discussion: Reducing the number of patients with the introduction of the DRG model with respect to reducing the number of patients at the national level where there has been a decline in the number of patients by $4 \%$. By reducing the number of hospitalized patients reduces the number of hospital days by $19.9 \%$. In a similar study conducted in Italy, decreased the average number of hospital days by $21.1 \%$. Survey concluded that reduces the average hospital stay of hospitalized patients in hospital Kochani (6.81 to 5.40 days). Nationwide reduction in the average hospital stay is from 6.25 to 5.9 days, from the research conducted in Italy showed a decline in the average hospital stay from 9.1 to 8.8 days, while in Croatia dropped the average hospital stay of 0.9 days. Kochani index hospital costs before introducing DRG is 107.7 or rising costs after the introduction of the DRG model is $7.7 \%$. Hospital costs are increasing with the introduction of the DRG in Gynecology and Obstetric Ward and Children's Ward, and reduced Surgical Ward internally and Neuropsychiatric Ward.

Conclusion The results of the survey show that the DRG model is a better way of paying for hospitals than the previous method of payment for completed services. In each segment of the tested clinical performance Kochani hospital after DRG introduction marks an improvement in the work of the hospital.

Recommendations The Public Health Institution General Hospital Kochani is required to develop its re sources conforming to the method of payment according to DRG. For further reduction of the duration of the hospital stay, the hospital days, the number of referrals to tertiary level, as well as the expenses of medical care an improvement of the working conditions is vital, by implementation of new procedures, new diagnostic methods, continual education of the medical personnel, modernization of the informatics equipment.

Key words: Diagnostic Related Groups (DRG); evaluation; duration of hospital stay; expenses; payment.

Mentor: Prof. Dr. Fimka Tozija.

Defended: 19.11.2012.

Natasha I. Stavreva. The quality of life in the persons with implanted oral-prostetic dentures in the persons aged 65 years and more in the Republic of Macedonia [MScPH thesis]. Skopje, Republic of Macedonia: Centre for Public Health, Faculty of Medicine, Ss Cyril and Methodius University of Skopje; 2013. OA Maced J Med Sci. 2013 Dec 15; 1(1):148-159.

Introduction: In the second half of the 20th century, health is becoming a global issue. Issue that affects in many basis and whose global solution is beyond the capacity of the individual or group of countries. It can not be talked about health, not to highlight an important component of overall health, and that is oral health. As well as general health, oral health affects different countries, different cultures, different sexual structures, and different age groups, but especially the adult population of people, including geriatric population, which is a specific category, whose number at the end of last century onwards is in increasing growth. Aging is conditioned by social, occupational, psychological, financial and physical changes. All of these directly affect health and health needs of the elderly. In the adult population (older than 65 years) due to their specific needs and disease significant changes in the structure of diseases occurs. In such conditions, health problems require new and rapid approaches to their resolution, and thus improving the quality of life. Health and health condition are essential components of quality of life of the overall population. Poor oral health and undermines the overall health. Difficulties in chewing and speech of adult patients with lost teeth lead to the need of incorporating oral-prosthetic dentures. Raising the general and health culture of the population and promotion of health, education of dental staff, introduction of new successful methods and materials, would lead to a reduction of the aforementioned oral problems, improving oral and general health and quality of life.

The main aim of this research is to assess the quality of life of institutionally sheltered geriatric population (persons aged 65 years and more), with built-in oral 
prosthetic dentures by determining the impact of oral health on daily activities, on the level of physical and psycho-social interactions.

Materials and methods: The survey was a prospective transversal (cross-sectional) study that was conducted among 165 institutionally sheltered patients aged over 65 years in the Gerontology Institute "13th November" - Skopje and 170 patients from the dental specialist clinics at age 65 and older. For statistical processing of data obtained during the survey data base was created in the statistical program SPSS for Windows. For testing the significance of the difference depending on the type and distribution of responses the following were used: non-parametrical tests (Chi-square, Yates corrected test, Fisher-exact test, Mann-Whitney test, Kruskal Wallis test), parametric tests (t-test for independent samples, Analysis of variance). The correlation between certain parameters were determined using the Pearson's coefficient of linear correlation and Spearmann's coefficient of rank correlation. All statistical tests were processed using the required statistical significance of $p<0,05$ (the results are considered statistically significant), and the results with a value of $p>0,05$ marked the absence of record for statistical significance

Results and discussion: The age of respondents at Gerontology Institute ranged from 65 to 92 years, while the age of patients from specialist dental clinics was between 65 and 87 years. The gender structure dominated by female with almost equal representation and 102 (61 .8\%) in institutional sheltered patients and $107(62.9 \%)$ at those of specialist dental clinics. The majority of institutional sheltered respondents are of Macedonian nationality (66.1\%), the Albanian ethnic community accounts for $14.5 \%$, and $3.64 \%$ are Romes, while from respondents from specialist dental clinics $62.9 \%$ were Macedonian, $12.9 \%$, Albanian and $8.2 \%$ are Romes. In terms of education, the highest percentages of respondents were with primary education in both groups (50.3\% vs $42.9 \%)$. Among respondents from the first group in the urban living environment are 89 (53.9\%), while in the second group the number and percentage were 78 (45.9\%). During clinical examination of the respondents regarding the type of built-oral prosthetic devices, two groups are dominated by patients with internal upper and lower total dentures (43.6\% vs $26.5 \%)$. The analyzed results obtained from answers to GOHAI questionnaire we could see that at the institutional sheltered elderly patients there is substantial difference in quality of life in terms of physical and psycho-social dimension, compared with adults from the dental specialist clinics.

Conclusion: From the analysis of the total score obtained using GOHAl indicator we came to the conclusion that in institutionally sheltered geriatric patients older than 65 years, the condition of oral health is unsatisfactory. This is indicated by the fact that the highest score is 40 and maximum score by
Licker-scale is 48. Quality of life in institutional sheltered patients shows substantial difference in terms of physical and psycho-social dimension compared to the patients from specialist dental clinics.

Key words: GOHAl indicator (questionnaire); oral health; geriatric population over 65 years; physical dimension; psychosocial dimension; quality of life; complete and partial dentures.

Mentor: Prof. Dr. Beti Zafirova-Ivanovska.

Defended: 28.11.2012.

Marko Shopov. Preventable factors of risk for cardiovascular diseases in the health workers of the primary health protection from the Skopje region - possibilities for public health action [MScPH thesis]. Skopje, Republic of Macedonia: Centre for Public Health, Faculty of Medicine, Ss Cyril and Methodius University of Skopje; 2013. OA Maced J Med Sci. 2013 Dec 15; 1(1):148-159.

Introduction: Despite a number of the risk factors for cardiovascular diseases (CVD) are potentially preventable, they still are a significant cause of morbidity and mortality worldwide.

Aim of the study: To assess the frequency of preventable risk factors for CVD in health care workers (HCW) employed in primary care settings, to compare the frequency of risk factors for CVD with their frequency in administrative workers (AW), as well as to compare the frequency of risk factors for CVD between physicians and nurses within the group of HCW.

Methods: We performed a cross-sectional study including $160 \mathrm{HCW}$ (80 doctors and 80 nurses) and $160 \mathrm{AW}$ employed in the health care sector. A specially designed questionnaire based on standardized questionnaires from this field and consisted from seven parts (demographics, family history for CVD, personal history for arterial hypertension, diabetes mellitus and hyperlipoproteinemia (HLP), body mass index (BMI), smoking status, physical activity, and stress) was completed by all examinees. The data obtained were statistically processed by descriptive and analytical methods.

Results: The frequency of arterial hypertension, diabetes mellitus, and HLP among HCW was $29.4 \%$, $8.1 \%$, and $25.0 \%$, respectively. There was no statistical significant difference in the frequency of arterial hypertension and diabetes mellitus between HCW and AW ( $p>0.05$ ), while the frequency of HLP was significantly higher in HCW as compared with $\mathrm{AW}$ $(p<0.05)$. The frequency of arterial hypertension was significantly higher in physicians compared with nurses $(p<0.05)$, while there was no significant difference in the frequency of diabetes mellitus and HPL between two groups of HCW ( $p>0.05)$. A BMI value higher than its normal value was registered in 
more than a half of the HCW $(54.8 \%)$. The mean BMI value did not differ significantly between $\mathrm{HCW}$ and AW, as well as between physicians and nurses $(p>$ $0.05)$. Approximately a half of the HCW $(47.5 \%)$ were daily smokers. The frequency of daily smokers among HCW did not differ significantly between HCW and AW, as well as between physicians and nurses $(p>$ $0.05)$. Majority of the HCW $(78.0 \%)$ reported exposure to tobacco smoke from other smokers in the household and/or in the workplace. There was not significant difference in the frequency of passive smokers between HCW and AW, as well as between physicians and nurses $(p>0.05)$. The frequency of HCW with no regular physical activity was $38.1 \%$. There was no significant difference in the frequency of the examinees with regular physical activity either between HCW, or between physicians and nurses ( $p$ $>0.05$ ). We hound a high frequency of stress indicators among HCW and a mean value of stress index in these workers was 6.25 (scale ranging from 1 to 10). The difference in mean value of stress index of HCW and AW, as well as of physicians and nurses, was statistically non-significant $(p>0.05)$. The regular preventive medical check-ups were not performed by majority of the HCW (65.6\%). There was no significant difference in the frequency of the examinees who did not perform regular preventive medical check-ups neither between HCW and HW, nor between physicians and nurses.

Conclusions: Our results indicate a high frequency of the preventable risk factors for CVD among HCW. The frequency of the preventable risk factors for CVD did not differ significantly between HCW and AW, as well as between doctors and nurses. These findings emphasis a need for public health action for reduction or elimination of preventable risk factors for CVD in HCW.

Key words: administrative workers; cardiovascular diseases; doctors; health care workers; nurses; preventable risk factors.

Mentor: Prof. Dr. Jordan Minov.

Defended: 27.12. 2012.

Urime Demire-Shaipi. Estimation of knowledge, attitudes and practice for the control of intrahospital infection and security in the work of doctors-stomatologists in the town Skopje, Republic of Macedonia [MScPH thesis]. Skopje, Republic of Macedonia: Centre for Public Health, Faculty of Medicine, Ss Cyril and Methodius University of Skopje; 2013. OA Maced J Med Sci. 2013 Dec 15; 1(1):148-159.

Introduction: Hospital Associated Infections (HAl) are infections occurring in a patient during the process of care in a hospital or other health-care facility, which was not present or incubating at the time of admission. HAl represent major challenge for dentistry. Patients and dental health care workers are exposed daily to various pathogens, including viral hepatitis (HBV, HCV), HIV, herpes viruses and Mycobacterium Tuberculosis. Hepatitis B and C viruses attack the liver and are major causes of cirrhosis and liver cancer. Associated with HIV, these diseases often end fatally. Proper application of Standard Precautions (SP), protects the dental health care workers and patients from $\mathrm{HAl}$ and reduce the risk of cross-infection and cross-contamination. As the rate of HIV caseload is on the rise, prevention of HIV and HBV in dentistry should be a concern in R. Macedonia as well.

Ohjectives: To assess the status of dental infection control and professional safety in city of Skopje, Republic of Macedonia.

Material and methods: This is a prospective cross sectional study. The study assesses the level of knowledge, attitudes and practice (KAP) regarding dental infection control and professional safety among dentists in private clinical practice and faculty members of Public Dental School in city of Skopje. For the purpose of this research a "Questionnaire on Dental Infection Control and Professional Safety" (Appendix I), with 97 variables was used. The Questionnaire was translated from English to Macedonian and was modified according to the needs of our population of interest. Statistical processing of the obtained data was analyzed using SPSS for Windows, version 13.0.

Results: From the total number of 188 dentists who completed the survey, $45.7 \%$ were practicing in the Public University Dental Clinic in city of Skopje, while $54.3 \%$ were practicing in the private dental offices. Total of $38.8 \%$ of respondents, were male and $61.2 \%$ were female dentists. Age of the respondents ranges from 24 to 64 years, with an average age of 40.6 (SD \pm 9.4). According to the results, only about $17 \%$ of dentists in both groups have written program for infection control in dentistry and protocol for Post Exposure Prophylaxis (PEP) in their workplace. Knowledge of dentists regarding the Standard Precautions are unsatisfactory because only about $50 \%$ of the respondents gave correct answers to questions of this group. Especially, there is an in sufficient knowledge regarding the sterilization of critical and semi-critical instruments (only $395 \%$ of faculty dentists and $41.2 \%$ of dentists in private clinics correctly answered that critical and semicritical instruments must both be sterilized). Knowledge about contaminants in dental water systems and the danger of infection is low as well $(43.02 \%$ vs. $26.5 \%$ answered affirmatively that the dental water system may be a potential reservoir of pathogens). Immunized were only $17.4 \%$ of the faculty dentists and $29.4 \%$ of dentists in private clinics. Dominant part of the respondents in both groups emphasize the need for mandatory training and annual updates on infection control and safety at work 
(90.7\% of faculty vs. $90.2 \%)$ private) and considers that measures for dental infections control is not waste of time. About $50 \%$ of dentists believe that they have sufficient knowledge and are properly trained to practice safe dentistry and only $27.9 \%$ faculty dentists and $18.6 \%$ of dentists in private clinics are familiar with their HIV status. High percentage of dentists $(90 \%)$ does not feel comfortable in treating patients with infectious diseases. They have negative attitude (stigmatization) toward them and around $80-85 \%$ use extra precautions while treating patients with infectious diseases. About $70 \%$ of dentists use double gloves during treatment of infectious patients, and schedule them for treatment on different days or time (79.1\% faculty vs. $72.55 \%$ private clinics). Respondents from both groups attended continuing education and training on dental infection control and safety in a very small percentage (around 20\%). Only $20.9 \%$ of the faculty dentists, compared to $75.9 \%$ dentists in private clinics had completed the mandatory preventive examinations. Faculty dentists significantly in higher percentage, compared to private dentists, reported lack of instruments and hand pieces (63.95\% vs. $20.6 \%$ ). Only $31.4 \%$ of faculty dentists and $62.75 \%$ of dentists in private clinics disinfected their impressions and less than $27 \%$ sterilize hand pieces between patients. The majority of them $(69.8 \%$ vs. $82.35 \%$ ) use dry heat for sterilization of instruments. Only around the half of the respondents use autoclaves. Eye protection is issued by $47.7 \%$ of faculty and $66.7 \%$ dentists in private clinics, and gloves are used by more than $90 \%$ of dentists in survey. The results from this current study were compared with similar studies carried out in other countries. It was concluded that dental infection control measures in the Republic of Macedonia are far behind Dental Standard Precautions implemented in developed countries such as USA, Canada, Australia, and the United Kingdom, but the results are similar with studies conducted in other developing countries.

Conclusions: Results of this study showed that there was an increased awareness and positive attitude towards training and education on infection control and dental safety but the level of knowledge, attitudes and practice of dental infection control between the faculty members and dentist who work in a private clinical practice, in city of' Skopje are similarly substandard. This highlights the need to develop national standards and protocols on dental safety, curriculums for dental students as well as mandatory continuing education on infection control in dentistry in the Republic of Macedonia.

Key words: Knowledge; Attitudes; Practices; Infection Control in Dentistry; occupational safety; HBV; HCV; HIV; dentists; stigma; Dental Skopje; Republic of Macedonia.

Mentor: Prof. Dr. Kakja Popovska-Jovanovska.

Defended: 27.12.2012. 